\title{
Identification of $\mathrm{H7}$ as a novel peroxiredoxin I inhibitor to induce differentiation of leukemia cells
}

\author{
Wei Wei ${ }^{1, *}$, Chunmin Ma ${ }^{1, *}$, Yang Cao ${ }^{1, *}$, Li Yang ${ }^{1, *}$, Zhimin Huang ${ }^{1, *}$, Dongjun Qin ${ }^{1}$, \\ Yingyi Chen ${ }^{1}$, Chuanxu Liu ${ }^{2}$, Li Xia ${ }^{1}$, Tongdan Wang ${ }^{1}$, Hu Lei ${ }^{1}$, Yun Yu ${ }^{1}$, Min Huang ${ }^{1}$, \\ Yin Tong ${ }^{4}$, Hanzhang $\mathrm{Xu}^{1}{ }^{1}$, Fenghou $\mathrm{Gao}^{3}$, Jian Zhang ${ }^{1, *}$, Ying-Li Wu ${ }^{1, *}$ \\ ${ }^{1}$ Hongqiao International Institute of Medicine, Shanghai Tongren Hospital/Faculty of Basic Medicine, Chemical Biology \\ Division of Shanghai Universities E-Institutes, Key Laboratory of Cell Differentiation and Apoptosis of The Chinese Ministry \\ of Education, Shanghai Jiao Tong University School of Medicine, Shanghai 200025, China \\ ${ }^{2}$ Department of Hematology, Xinhua Hospital Shanghai Jiao-Tong University School of Medicine, Shanghai, 200092, China \\ ${ }^{3}$ Institute of Oncology, Shanghai 9th People's Hospital, Shanghai Jiao Tong University School of Medicine, Shanghai 200011, \\ China \\ ${ }^{4}$ Department of Hematology, Shanghai First People's Hospital, Shanghai Jiao-Tong University School of Medicine, Shanghai, \\ 200081, China \\ *These authors contributed equally to this work
}

Correspondence to: Ying-Li Wu, e-mail: wuyingli@shsmu.edu.cn Jian Zhang, e-mail: jian.zhang@sjtu.edu.cn

Keywords: peroxiredoxin, leukemia, cell differentiation, reactive oxygen species

Received: June 09, 2015

Accepted: November 28, 2015

Published: December 26, 2015

\section{ABSTRACT}

Identifying novel targets to enhance leukemia-cell differentiation is an urgent requirment. We have recently proposed that inhibiting the antioxidant enzyme peroxiredoxin I (Prdx I) may induce leukemia-cell differentiation. However, this concept remains to be confirmed. In this work, we identified $\mathrm{H7}$ as a novel Prdx $I$ inhibitor through virtual screening, in vitro activity assay, and surface plasmon resonance assay. Cellular thermal shift assay showed that $\mathrm{H7}$ directly bound to Prdx $\mathrm{I}$ but not to Prdxs II-V in cells. $\mathrm{H} 7$ treatment also increased reactive oxygen species (ROS) level and cell differentiation in leukemia cells, as reflected by the upregulation of the cell surface differentiation marker CD11b/CD14 and the morphological maturation of cells. The differentiation-induction effect of $\mathrm{H7}$ was further observed in some non-acute promyelocytic leukemia (APL) and primary leukemia cells apart from APL NB4 cells. Moreover, the ROS scavenger $\mathrm{N}$-acetyl cysteine significantly reversed the H7-induced cell differentiation. We demonstrated as well that H7-induced cell differentiation was associated with the activation of the ROS-Erk1/2-C/EBP $\beta$ axis. Finally, we showed $\mathrm{H7}$ treatment induced cell differentiation in an APL mouse model. All of these data confirmed that Prdx I was novel target for inducing leukemia-cell differentiation and that $\mathrm{H7}$ was a novel lead compound for optimizing Prdx I inhibition.

\section{INTRODUCTION}

In acute myeloid leukemia (AML), immature cells are blocked abnormally at an early stage of their development and fail to differentiate into functional mature cells. The introduction of all trans-retinoic acid into the acute promyelocytic leukemia (APL) therapy validated the concept of differentiation therapy [1]. However, differentiation therapy remains limited to APL treatment. Despite the discovery of many differentiation agents, such as vitamin $\mathrm{D}$ and 1,25-dihydroxy vitamin D3 [2-4], differentiation therapy failed in most non-APL leukemia cases. Therefore, a deep understanding of the molecular mechanisms of leukemia cell differentiation, as well as identifying novel differentiation agents, is urgently required.

In the mammalian hematopoietic system, the reactive oxygen species (ROS) levels are apparently important in controlling myeloid precursor differentiation. Accumulating data have shown that ROS-modulating agents, such as 
arsenic trioxide, DFO, and tetrandrine, may regulate leukemia differentiation by increasing ROS levels [5-10]. Therefore, identification of small molecules exhibiting ROS-modulating activity may provide new tool in investigating the underlying mechanisms of leukemia-cell differentiation and in developing new therapy for leukemia.

Peroxiredoxins (Prdxs) are a superfamily of small non-selenoperoxidases that contain essential catalytic cysteine residues and use thioredoxin as an electron donor in reducing hydrogen peroxide $\left(\mathrm{H}_{2} \mathrm{O}_{2}\right)$ [11]. At least six mammalian isoforms, including two-cysteine (Prdxs I-IV), atypical two-cysteine ( $\operatorname{Prdx} \mathrm{V})$, and one-cysteine (Prdx VI) Prdxs have been identified [12-13]. Prdx I is abundant in cells, constituting a total of $0.2 \%-1 \%$ of soluble protein in cultured mammalian cells [14]. Emerging data have shown that Prdx I levels are elevated in several human cancer cells and tissues, such as oral, esophageal, pancreatic, follicular thyroid, breast, and lung cancers. The elevated Prdx I proteins enhance the aggressive survival phenotype of cancer cells and confer an increased resistance to chemotherapy and radiotherapy [15-19]. We recently demonstrated that adenanthin, a natural diterpenoid, could induce differentiation of leukemia cells through the Prdx I/ROS axis [20-21]. We then proposed that Prdx I represents a novel target in

$\mathbf{A}$

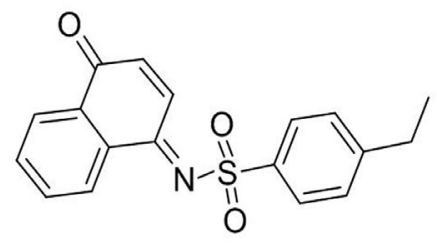

$\mathbf{C}$

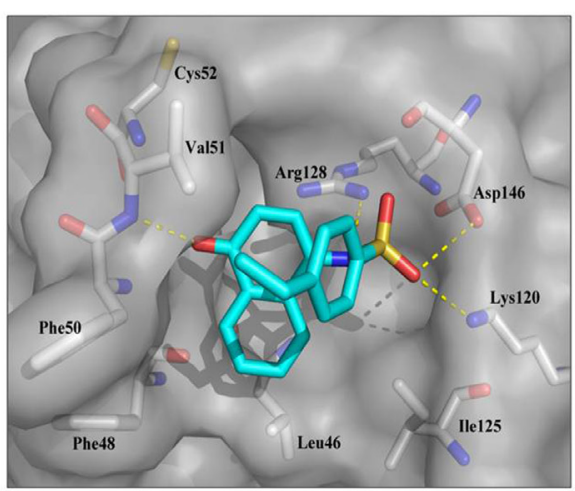

developing differentiation agents. However, this concept need to be further confirmed.

Using virtual screening, along with in vitro and cellular assay, we identified in this study that $\mathrm{H} 7$ is a novel Prdx I inhibitor. We further demonstrated that $\mathrm{H} 7$ induces leukemia-cell differentiation in vitro and in vivo. We validate the notion that Prdx I is a novel target that induces leukemia-cell differentiation and $\mathrm{H} 7$ is a novel lead compound for developing Prdx I inhibitors.

\section{RESULTS}

\section{H7 is a novel Prdx I inhibitor}

We used virtual screening, along with in vitro Prdx I activity assay, to identify the novel Prdx I inhibitors. In the virtual screening, the candidate compounds from different scaffolds were selected and their potency for Prdx I inhibition was analyzed using the in vitro Prdx I activity assay. Among the compounds, H7 (Figure 1A) showed the most potent inhibition of Prdx I activity and was thus selected for further investigation. The $\mathrm{IC}_{50}$ of $\mathrm{H} 7$ on Prdx I activity was $7.85 \mu \mathrm{M}$ (Figure 1B). Moreover, docking study showed that $\mathrm{H} 7$ is buried in a pocket composed of Leu46, Phe48, Phe50, Val51, Cys52, Lys120,

B

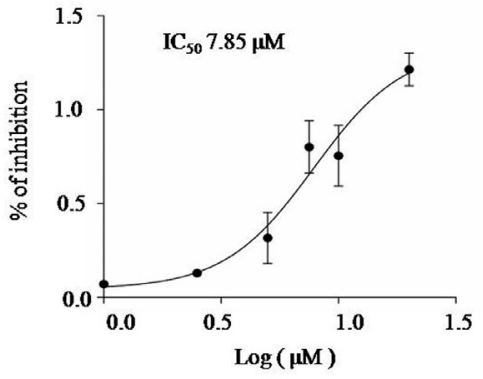

D

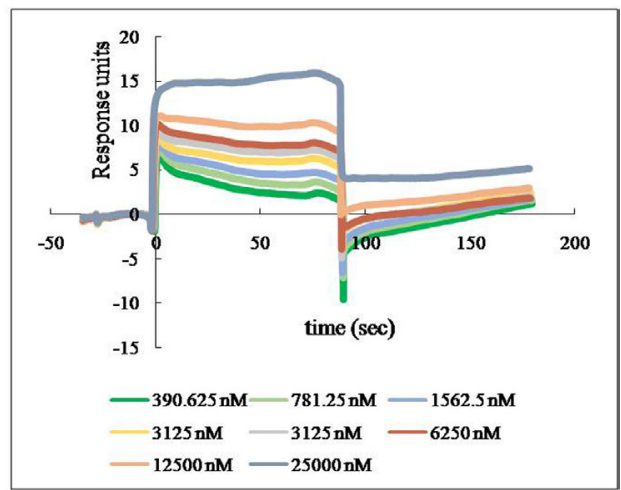

Figure 1: H7 inhibits Prdx I catalytic activity. (A) Chemical structure of H7. (B) The recombinant Prdx I protein was incubated with the indicated concentration of $\mathrm{H} 7$ for $1 \mathrm{~h}$, and its peroxidase activity was monitored for $1200 \mathrm{~s}$. The $\mathrm{IC}_{50}$ of $\mathrm{H} 7$ on Prdx I was calculated by the Graphpad prism software. All values represent the means \pm S.D. of three independent experiments. (C) Binding model of $\mathrm{H} 7$ and Prdx I. The molecular surface of Prdx I protein is shown in gray and the H7 molecule is shown as sticks with light blue carbons. (D) SPR analysis of the binding between Prdx I and H7. The recombinant Prdx I protein was immobilized on an activated CM5 chip. H7 was then flowed across the chip at increasing concentrations. The chip was regenerated between concentrations using $2 \mathrm{M}$ glycine. Dose-dependent binding of $\mathrm{H} 7$ was observed across the concentration range. 
Ile125, Arg128, and Asp146. Moreover, The sulfonyl and carbonyl group of $\mathrm{H} 7$ form four hydrogen bonds, of which make it to stably interact with and inhibit Prdx I, with both side chains of Lys120, Arg128, Asp146 and main chain of Val51, respectively (Figure 1C). These data suggest that H7 is a novel Prdx I inhibitor.

The binding between H7 and Prdx I was further evaluated by surface plasmon resonance (SPR) assay using a biacore platform. The sensorgrams showed that $\mathrm{H} 7$ rapidly associated and disassociated from the immobilized Prdx I at a dissociation constant of $1.57 \mu \mathrm{M}$ (Figure 1D). Moreover, the response signal during the dissociation phase returned to the baseline level for $\mathrm{H} 7$, indicating complete dissociation of the compound from Prdx I. These data suggest that H7 is non-covalently bound to Prdx I.

\section{H7 interacts with Prdx I in cells}

To investigate whether the interaction between H7 and Prdx I observed in vitro does occur in cells, we performed cellular thermal shift assay (CETSA). CETSA is a newly developed method of measuring the direct binding of protein with its ligand in cells; this technique is based on the concept that the direct binding of a small molecule to its target protein may increase the stability of proteins in response to heat [22]. Figure 2A and 2B showed that the addition of $\mathrm{H} 7$ but not DMSO into the cell lysates increased the stability of Prdx I at different temperatures. Prdx I was highly stabilized at $75.9^{\circ} \mathrm{C}$. However, $\mathrm{H} 7$ did not significantly affect the stability of Prdxs II-V, indicating the relative selectivity of $\mathrm{H} 7$ on Prdx I (Figure 2C). Moreover, the stabilization effect of $\mathrm{H} 7$ on Prdx I is dose dependent (Figure 2D). Given that Prdx I functions as a $\mathrm{H}_{2} \mathrm{O}_{2}$ scavenger, we subsequently determined whether $\mathrm{H} 7$ treatment increases ROS level in NB4 cells. The ROS level in the H7-treated NB4 cells gradually increased, peaked after $12 \mathrm{~h}$, and then declined after $24 \mathrm{~h}$ (Figure 4E). These data demonstrate that H7 could reach its target protein Prdx I in a biologically relevant setting, leading to increased ROS level.

\section{H7 induces NB4 cell differentiation}

Given that targeting Prdx I by adenanthin induces leukemia-cell differentiation [20], we then determined whether $\mathrm{H} 7$ can also induce leukemia-cell differentiation. H7
$\mathbf{A}$

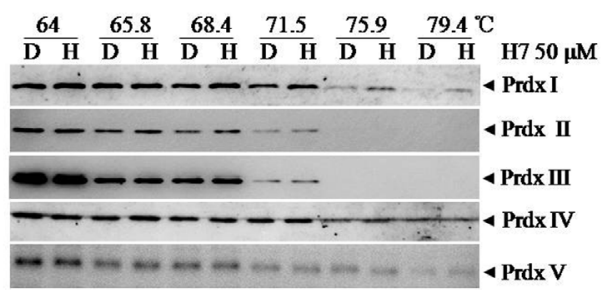

C

D

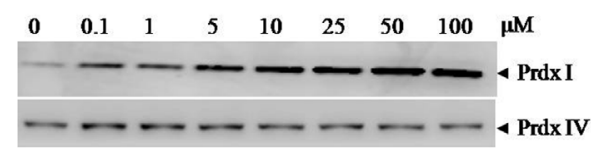

C

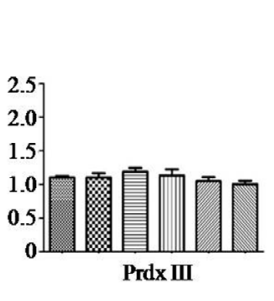

B

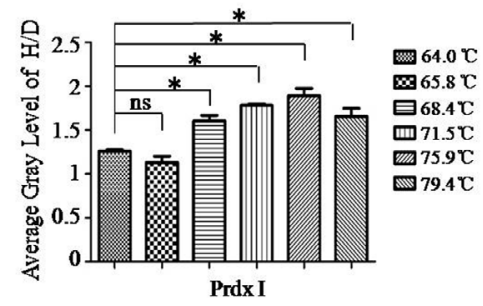

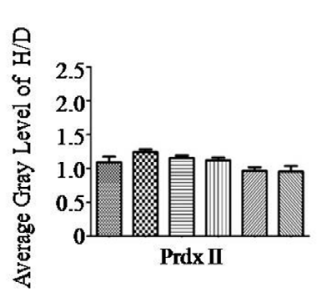

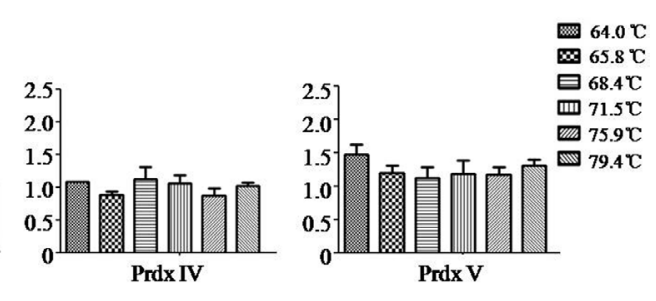

$\mathbf{E}$

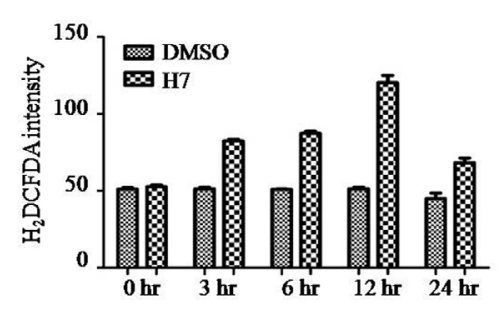

Figure 2: H7 interacts with Prdx1 in cells. (A) The NB4 cell lysate was incubated with DMSO or H7 at different temperatures for 3 min followed by centrifugation at $20000 \mathrm{~g}$. The supernatant was subjected to Western blot analysis of the Prdxs I-V proteins. (B-C) The intensity of the bands of the Prdxs I-V proteins were quantified by the Quantity One software, and the relative ratio of H7- and DMSOtreated bands (H / D) was displayed. All values represent the means \pm S.D. of three independent experiments. ${ }^{*} p<0.05$. Prdx IV was used as a loading control. (D) The NB4 cell lysate was incubated with DMSO or different $\mathrm{H} 7$ concentrations at $75.9^{\circ} \mathrm{C}$ for 3 min followed by centrifugation at 20,000 g. The supernatant was subjected to Western blot analysis for the Prdx I protein. Prdx IV was used as a loading control. (E) The NB4 cells were treated with H7 for different times, and the intracellular ROS were evaluated by DCFDA staining. Each experiment was repeated at least three times. 
exerts dose- and time-dependent growth inhibition effect on NB4 cells (Figure 3A). At $4 \mu \mathrm{M}, \mathrm{H} 7$ significantly inhibited NB4 cell growth without obvious loss of cell viability. Thus, we selected this $\mathrm{H} 7$ concentration in subsequent experiments. The NB4 cells were treated with $\mathrm{H} 7$ for 24,48 , and $72 \mathrm{~h}$, and then cell differentiation was monitored. $\mathrm{H} 7$ treatment increased the percentages of CD11b- and CD14positive cells (Figure 3B, 3C); morphologically, H7 also reduced the nuclei/cytoplasm ratio, indicating monocyte differentiation of NB4 cells (Figure 3D).

\section{Prdx I is essential in H7-induced leukemia-cell differentiation}

To further confirm the role of Prdx I in H7-induced cell differentiation, Prdx I was knocked down (Figure 4A, NB4 $4^{\text {shPrdx I})}$ or overexpressed in NB4 cells (Figure $4 \mathrm{C}$, NB4 $\left.{ }^{\text {Prdx I }}\right)$, and nonspecific shRNA- $\left(\mathrm{NB} 4^{\text {shNC }}\right)$ or control vector-transfected cells (NB4 ${ }^{\text {vector }}$ ) were used as negative controls, respectively. Prdx I knockdown induced cell differentiation in NB4 cells, whereas H7 treatment further increased the percentages of CD11b/CD14-positive cells (Figure 4B, Supplementary Figure S2A). By contrast, Prdx I overexpression in NB4 cells partially blocked the differentiation effect of H7 (Figure 4D, Supplementary

$\mathbf{A}$

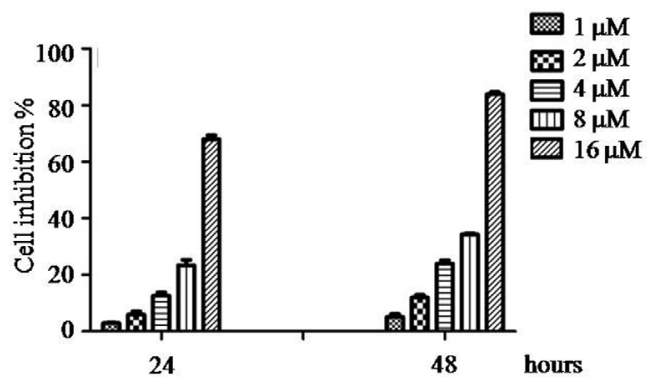

$\mathbf{B}$

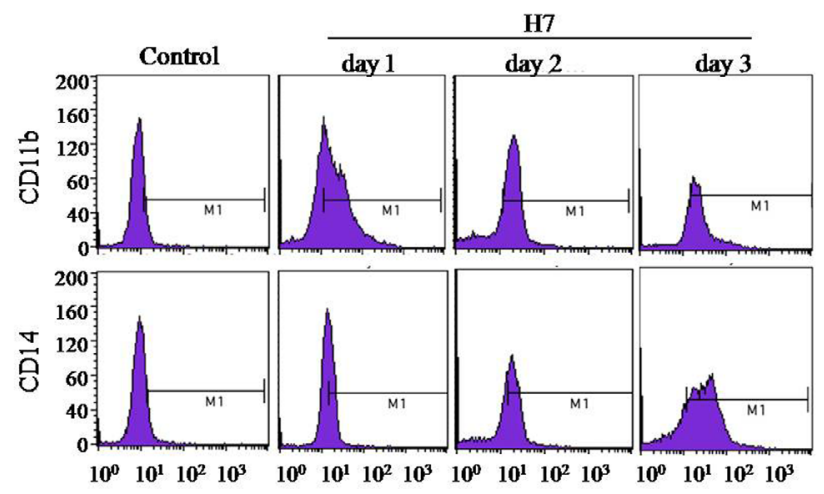

Figure S2B). These data suggest that Prdx I plays an essential role in $\mathrm{H} 7$-induced cell differentiation.

\section{H7 induces cell differentiation of non-APL leukemia cell lines and primary cells}

To investigate whether the differentiation-inducing effect of H7 is limited to NB4 cells, we investigated the leukemia cell lines U937 and HL60, the primary leukemia cells, and the normal cord blood or bone marrow mononuclear cells (CBMNCs or BMMNCs). U937, HL60, and NB4 cells expressed high protein level of Prdx I. Varied expression levels of Prdx I were observed in primary leukemia cells. In addition, Prdx I was lowly expressed in normal CBMNCs and BMMNCs (Figure 5A). $\mathrm{H} 7$ treatment also induced cell differentiation in U937 (Figure 5B, 5C, Supplementary Figure S3A), in HL60 (Figure 5D, 5E, Supplementary Figure S3B), in some primary leukemia cells (Figure 5F, 5G, Table 1, Supplementary Figure S3C, 3D, 3E), and slightly in CBMNCs and BMMNCs (Table 1, Supplementary Figure S3F, 3G), as shown by the increased CD11b and CD14 expression and/or by the morphological changes in cells. These data suggest that the differentiation-inducing effect of $\mathrm{H7}$ is not limited to APL cells.

C

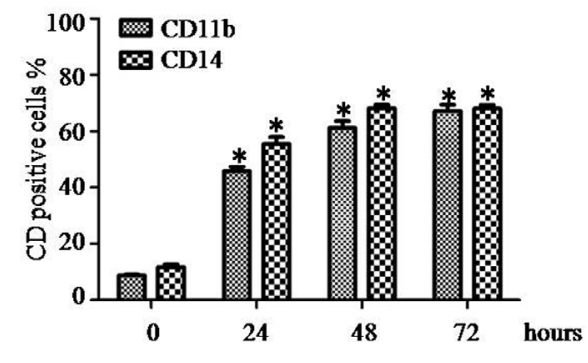

D

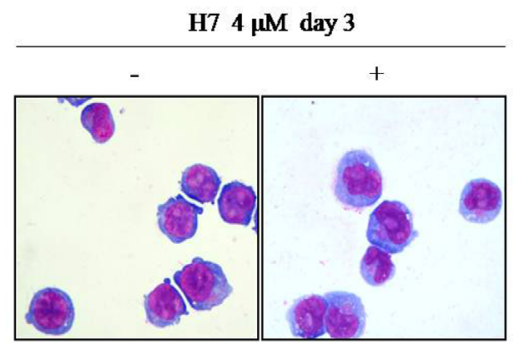

Figure 3: H7 induces partial differentiation of NB4 cells. (A) The NB4 cells were treated with different H7 concentrations for 24 and $48 \mathrm{~h}$. Cell proliferation was determined by trypan blue exclusion assay. (B-C) The NB4 cells were treated with H7 for 1, 2, and 3 days, and the CD11b and CD14 expression levels were determined by FACS. The representative histogram and percentages of CD11b and CD14 are shown in (B) and (C), respectively. All values represent the means \pm S.D. of three independent experiments. ${ }^{*} p<0.05$ compared with the control group. (D) The NB4 cells were treated with H7 for 3 days, and cell morphology was examined by Wright's staining and light microscopy $(100 \times)$. Each experiment was repeated at least three times. 


\section{H7 induces differentiation through activation of the ROS-Erk1/2-C/EBPß pathway}

Given that the Prdx I inhibitor adenanthin induces leukemia-cell differentiation by increasing ROS level, we hypothesized that $\mathrm{H} 7$ induces cell differentiation in a similar manner. To determine the role of ROS in H7-induced cell differentiation, we treated the NB4 cells with $\mathrm{H} 7$ in the presence or absence of N-acetyl cysteine (NAC), a ROS scavenger. NAC treatment significantly inhibited $\mathrm{H} 7$-induced CD11b and CD14 upregulation (Figure 6A). These data suggest that $\mathrm{H} 7$ induces leukemia-cell differentiation in a ROS-dependent manner.

Furthermore, activation of the AKT or MAPK signaling pathway has been implicated in ROS-mediated cell differentiation [23-24]. H7 treatment indeed activated AKT, as evidenced by the increased $\mathrm{AKT}^{\text {Ser473 }}$ phosphorylation (Figure 6B). However, knockdown of AKT expression by shRNA did not affect $\mathrm{H} 7$-induced cell differentiation, indicating that AKT activation is not involved in $\mathrm{H} 7$-induced cell differentiation (Supplementary Figure S4). Given that Erk1/2 activation is involved in adenanthin-induced differentiation, we then evaluated the possible role of Erk1/2. H7 treatment activated Erk1/2 (Figure 6B). By contrast, co-treatment of $\mathrm{H} 7$ with the Erk1/2 inhibitor PD98058 inhibited the H7-induced Erk1/2 activation (Figure 6C) and the CD11b

A

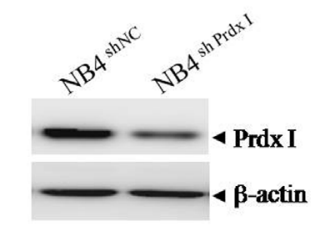

B

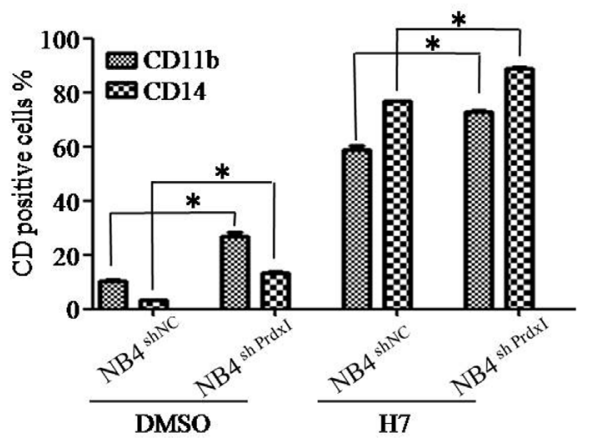

and CD14 upregulation (Figure 6D). These data suggest that Erk1/2 activation is involved in $\mathrm{H} 7$-induced cell differentiation.

$\mathrm{C} / \mathrm{EBP} \beta$ is also speculated to drive immature cells to become granulocytes and monocytes and has been shown as an important downstream target of Erk1/2 [25]; thus, we determined the $\mathrm{C} / \mathrm{EBP} \beta$ expression upon $\mathrm{H} 7$ treatment. $\mathrm{H} 7$ treatment increased $\mathrm{C} / \mathrm{EBP} \beta$ expression, which was abrogated by co-treatment of NAC (Figure 6B). The Erk1/2 inhibitor PD98059 also inhibited the H7-induced $\mathrm{C} / \mathrm{EBP} \beta$ upregulation (Figure 6C). Moreover, knockdown of C/EBP $\beta$ (Figure 6E) abrogated the $\mathrm{H} 7$-induced CD11b and CD14 upregulation (Figure 6F). These data suggest that $\mathrm{H} 7$-induced cell differentiation is mediated through the ROS-Erk1/2-C/EBP $\beta$ axis.

\section{H7 induces leukemia-cell differentiation in vivo}

Given that $\mathrm{H} 7$ induces cell differentiation in vitro, we then attempted to determine the effect of $\mathrm{H} 7$ in vivo in our APL mouse model. Compared with the vehicletreated leukemic mice, the intraperitoneal injection of $\mathrm{H} 7$ at 10 and $20 \mathrm{mg} / \mathrm{kg}$ increased the terminally differentiated cells in the bone marrow (BM) and peripheral blood (PB), as reflected by the increase in maturating myeloid cells showing differentiation-related morphologic features, such as condensed chromatin with indented, distorted, and

$\mathbf{C}$

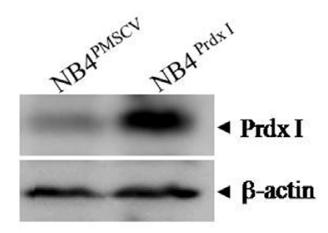

D

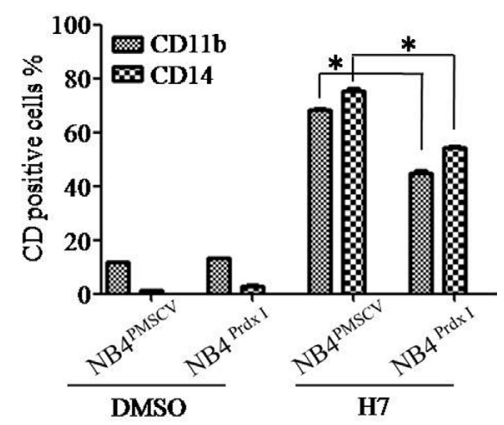

Figure 4: Knockdown or overexpression of Prdx I increases or decreases H7-induced cell differentiation. (A) The NB4 cells were transfected with non-specific shRNA (NB4 $4^{\text {shNC }}$ ) or Prdx I specific shRNA (NB4 $4^{\text {shrdx }}$ I). The indicated proteins were examined by Western blot. (B) The NB4 $4^{\text {shNC }}$ and NB4 $4^{\text {shPrdx } ~}$ cells were treated with $\mathrm{H} 7$ for 3 days, and the expression of CD11b, CD14 were determined by FACS. All values represent the means \pm S.D. of three independent experiments. ${ }^{*} p<0.05$ compared with the control group. (C) The NB4 cells were transfected with the vector (NB4 ${ }^{\mathrm{NC}}$ ) or Prdx I plasmid (NB4 ${ }^{\mathrm{Prdx}}$ I). The indicated proteins were examined by Western blot. (D) The NB4 ${ }^{\mathrm{NC}}$ and NB4 $4^{\mathrm{Prdx}} \mathrm{I}$ cells were treated with $\mathrm{H} 7$ for 3 days, and the expression of $\mathrm{CD} 11 \mathrm{~b}$ and $\mathrm{CD} 14$ were determined by FACS. All values represent the means \pm S.D. of three independent experiments. ${ }^{*} p<0.05$ compared with the control group. 
horse-shoe- or donut-shaped nuclei (Figure 7A). Moreover, we measured the mouse granulocytic differentiationrelated antigens Gr-1 and Mac-1 after gating for myeloid cells by FSC and SSC on flow cytometry. The results showed that in these myeloid cells, the percentages of Gr- $1^{+}$and $\mathrm{Mac}-1^{+}$cells were much higher in the BM of the H7-treated leukemic mice than those of the vehicle-treated leukemic mice (Figure 7B and 7C). These data indicate that $\mathrm{H} 7$ induces leukemia-cell differentiation in vivo.

\section{DISCUSSION}

This study found $\mathrm{H} 7$ as a novel Prdx I-inhibiting compound and demonstrated that H7 could induce leukemia-cell differentiation. These results have validated that Prdx I is a potential target in inducing leukemia-cell differentiation.

Since the discovery of ROS, the primary focus has been directed to the oxidative damage of biological macromolecules, including proteins, DNA, and lipids. However, accumulating evidence has suggested that changing the cellular ROS level is useful in altering the cell differentiation status $[6,26]$. The homeostasis of redox reactions in cells is controlled primarily by antioxidant enzymes, such as catalase, glutathione peroxidase 1, and Prdxs. We recently proposed that targeting the Prdx/ROS axis is a potential novel strategy in inducing leukemia-cell differentiation [20]. However, this hypothesis has not yet been confirmed.

To validate this hypothesis, we first attempted to identify the novel Prdx I inhibitor. We demonstrated that $\mathrm{H} 7$ is a novel Prdx I inhibitor, and this finding is supported by several lines of evidence: (A) H7 inhibits Prdx I activity in vitro. Moreover, our SPR analysis showed that $\mathrm{H} 7$ rapidly associated and disassociated with Prdx I, indicating their non-covalent bonding. The docking study also showed that $\mathrm{H} 7$ stably interacted with Prdx I through four hydrogen bonds. (B) H7 binds to Prdx I in cells. Compared with other methods used in confirming the interaction of small molecular compounds with proteins, CETSA can be performed more easily and can directly measure whether a drug molecule reached its targets in cells and animal models [27]. Using this method, we demonstrated that $\mathrm{H} 7$ indeed binds to Prdx $\mathrm{I}$ in a biologically relevant circumstance. However, H7 could not interact with the four other two-cystein Prdxs (Prdxs II-V), indicating that $\mathrm{H} 7$ is relatively selective to Prdx I. The interaction of H7 with Prdx I is further

$\mathbf{A}$

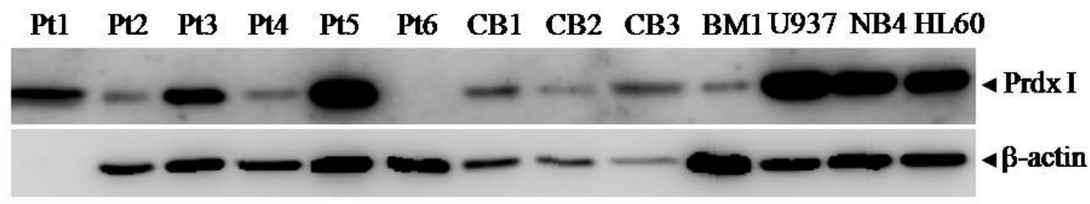

B

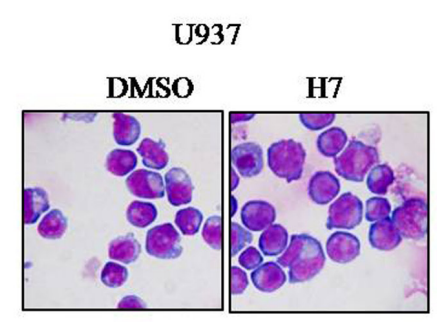

C

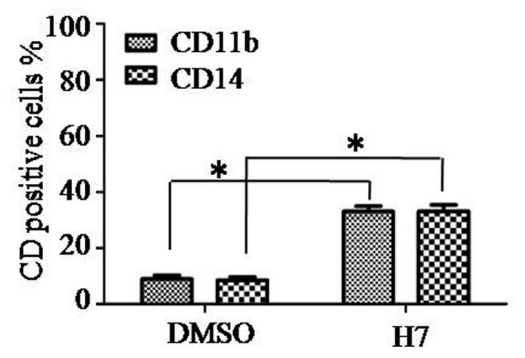

D

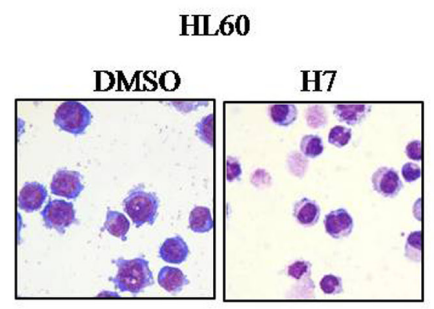

E

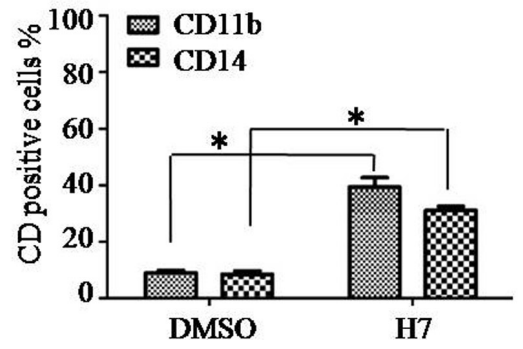

$\mathbf{F}$

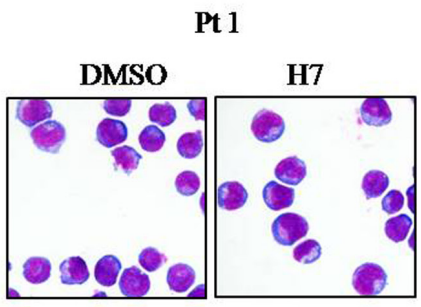

G

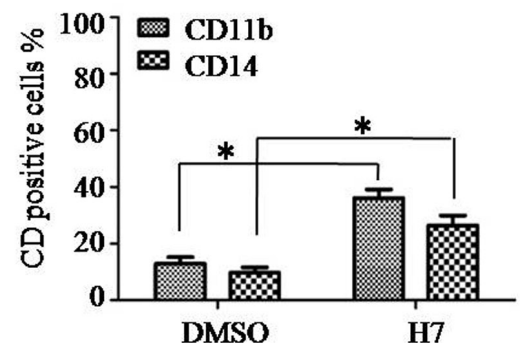

Figure 5: H7 induces monocyte differentiation in U937, HL60, and primary leukemia cells. (A) Prdx I expression in different cell lines and primary cells were determined by Western blot. (B-F) The indicated cells were treated with $\mathrm{H} 7$ for 3 days, and cell morphology was examined by Wright's staining and light microscopy $(100 \times)(\mathrm{B}, \mathrm{D}, \mathrm{F}$, day 3$)$. The cell surface markers CD11b and CD14 were detected by FACS, and the percentages of CD11b and CD14 are shown in (C, E, and G). All values represent the means \pm S.D. of three independent experiments. ${ }^{*} p<0.05$ compared with the control group. 
Table 1: Effect of $\mathrm{H7}$ on differentiation of primary blood cells

CD11b

CD14

\begin{tabular}{|c|c|c|c|c|}
\hline \multicolumn{2}{c}{ DMSO } & H7 & DMSO & H7 \\
\hline $12.62 \pm 1.45$ & $38.69 \pm 1.37^{*}$ & $8.54 \pm 0.57$ & $29.57 \pm 1.45^{*}$ \\
\hline $8.82 \pm 0.20$ & $35.48 \pm 0.82^{*}$ & $10.64 \pm 0.17$ & $40.78 \pm 0.39^{*}$ \\
\hline $7.63 \pm 0.16$ & $39.08 \pm 0.13^{*}$ & $11.4 \pm 3.91$ & $17.23 \pm 1.72$ \\
\hline $31.56 \pm 0.52$ & $43.76 \pm 1.87$ & $30.90 \pm 0.23$ & $48.15 \pm 0.24^{*}$ \\
\hline $38.56 \pm 0.18$ & $38.90 \pm 0.17$ & $23.31 \pm 0.56$ & $23.82 \pm 0.92$ \\
\hline
\end{tabular}

Note: Mononuclear cells from bone marrow or cord blood cells were treated with $\mathrm{H} 7$ for 3 days. The percentages of CD11b and CD14 were evaluted by FACS. All values represent means \pm S.D. of three independent experiments. ${ }^{*} p<0.05$ compared with the DMSO-treated cells.

demonstrated by drug affinity responsive target stability (DARTS) assay, which is another established and valid methodology for identifying and studying protein-ligand interactions. Indeed, H7 can protect Prdx I from proteaseinduced degradation, indicating that $\mathrm{H} 7$ may bind to Prdx I (Supplementary Figure S1). (C) Consistent with its Prdx I inhibition activity, $\mathrm{H} 7$ treatment increases ROS level in cells.

Using $\mathrm{H} 7$ as a chemical probe, we further demonstrated that targeting the Prdx I indeed induced leukemia-cell differentiation. Manipulating the protein level by overexpression or knockdown experiments could decrease or increase $\mathrm{H} 7$-induced cell differentiation, further demonstrating that Prdx I is critical in $\mathrm{H} 7$-induced cell differentiation. Consistent with our previous report [20], inducing cell differentiation by targeting Prdx I is not limited to NB4 cells. Other non-APL cells and primary leukemia blast cells could also be induced by $\mathrm{H} 7$ to differentiate. In addition, $\mathrm{H} 7$ treatment could induce cell differentiation in leukemic mice, although $\mathrm{H} 7$ failed to prolong the survival of leukemic mice possibly because of its toxicity. Injection of $\mathrm{H} 7$ at $20 \mathrm{mg} / \mathrm{kg}$ through the tail vein caused mummification necrosis of the tail. By contrast, the mice were more tolerant to intraperitoneal injection of $\mathrm{H} 7$, although long-term treatment at $20 \mathrm{mg} / \mathrm{kg}$ still resulted in early death of the leukemic mice (data not shown). Decreasing the concentration of $\mathrm{H} 7$ to $10 \mathrm{mg} / \mathrm{kg}$ will not only reduce its toxicity but also its efficacy. Therefore, optimizing $\mathrm{H} 7$ to reduce its toxicity is required. Taken together, these results further confirmed that targeting Prdx I could induce differentiation in APL and non-APL cells.

We previously demonstrated that the Prdx I-ROS axis is vital to the adenanthin-induced cell differentiation [20]. Consistent with this finding, NAC, a blocker of ROS, also inhibited $\mathrm{H} 7$-induced leukemia-cell differentiation. This observation suggests that $\mathrm{H} 7$ induces leukemia-cell differentiation through the Prdx I-ROS axis. Moreover, the activation of AKT or MAPK signaling pathway has been implicated in ROS-mediated cell differentiation [23-24]. H7 treatment activated AKT and Erk1/2.
However, inhibition of Erk1/2 but not of Akt could inhibit $\mathrm{H} 7$-induced cell differentiation, indicating that Erk 1/2 activation is involved in the $\mathrm{H} 7$-induced cell differentiation. $\mathrm{C} / \mathrm{EBP} \beta$ is also a key transcription factor regulating monocytic gene expression. Several reports and ours have shown that $\mathrm{C} / \mathrm{EBP} \beta$ is an important downstream molecule of Erk1/2 [28-29]. We then investigated the possible role of $\mathrm{C} / \mathrm{EBP} \beta$ in $\mathrm{H} 7$-induced leukemia-cell differentiation. As expected, NAC blocked the $\mathrm{H} 7$-induced $\mathrm{C} / \mathrm{EBP} \beta$ upregulation in leukemia cells. In addition, silencing of the $\mathrm{C} / \mathrm{EBP} \beta$ protein could weaken the ability of $\mathrm{H} 7$ to induce leukemia-cell differentiation. Thus, we proposed that $\mathrm{H} 7$ induces leukemia-cell differentiation through the Prdx I-ROS-Erk1/2-C/EBP $\beta$ axis.

In conclusion, our findings verified in principle that Prdx I is a novel target for inducing leukemia-cell differentiation. Moreover, H7 is a novel lead compound for optimizing Prdx I inhibition.

\section{MATERIALS AND METHODS}

\section{Virtual screening}

Virtual screening and docking were performed using Glide version 5.5 (Schrodinger Suite 2009) with default docking parameter settings. The Prdx I structure was retrieved from the Protein Data Bank (PDB entry: 1QQ2). Hydrogen atoms and charges were added during a brief relaxation, which was performed using the "Protein Preparation" module in Maestro and executing the "preparation and refinement" option; the restrained partial minimization was terminated when the root-mean-square deviation reached a maximum value of $0.3 \AA$ to relieve the steric clashes of amino acid residues located within $14 \AA$ from the catalytic thiolate of Cys52; these amino acid residues were defined as part of the binding site for docking studies. All crystallographic water molecules were removed from the coordinate set. Moreover, the compound library for screening was obtained from SPECS company [http://www.specs.net/ (October 1, 2013)]. All 180,000 compounds were desalted, neutralized, and 
$\mathbf{A}$

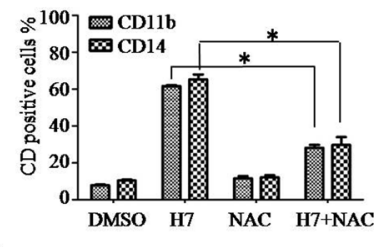

B

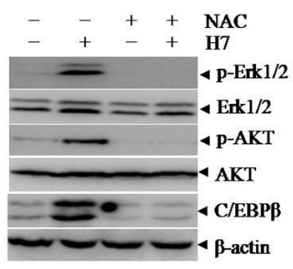

$\mathbf{C}$

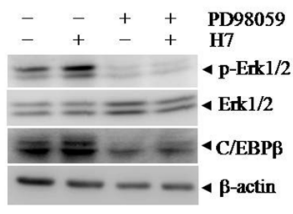

D

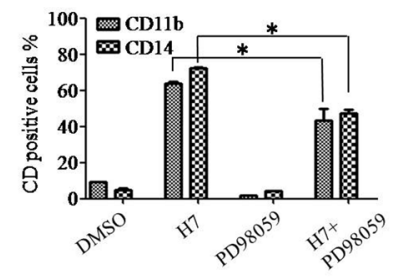

E

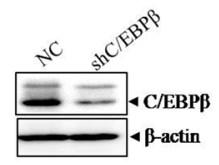

$\mathbf{F}$

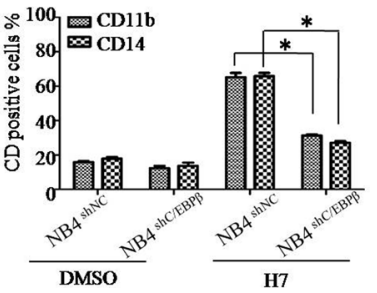

Figure 6: $\mathbf{H 7}$ induces monocyte differentiation via ROS-C/EBP $\boldsymbol{\beta}$ axis in NB4 cells. (A-B) The NB4 cells were treated with H7 in the presence or absence of NAC for 3 days, and the expression of the cell surface markers CD11b and CD14 was detected by FACS. All values represent the means \pm S.D. of three independent experiments. ${ }^{*} p<0.05$ compared with the single treatment (A); the indicated proteins were examined by Western blot (B). (C-D) The NB4 cells were treated with H7 for 3 days in the presence or absence of PD98059, and the expression levels of the cell surface marker CD11b and CD14 were detected by FACS. All values represent the means \pm S.D. of three independent experiments. ${ }^{*} p<0.05$ compared with the $\mathrm{H} 7$ treatment (D); the indicated proteins were examined by Western blot (C). (E-F) The C/EBP $\beta$ knockdown NB4 cells $\left(\mathrm{NB} 4^{\text {shC/EBP }}\right)$ and the control NB4 cells $\left(\mathrm{NB} 4^{\text {shNC }}\right)$ were treated with $\mathrm{H} 7$ for 3 days, and the

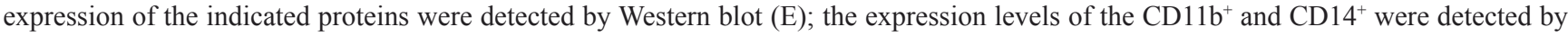
FACS. All values represent the means \pm S.D. of three independent experiments. ${ }^{*} p<0.05$ compared with the H7-treated NB4 ${ }^{\text {shNC }}$ cells $(\mathrm{F})$.

A

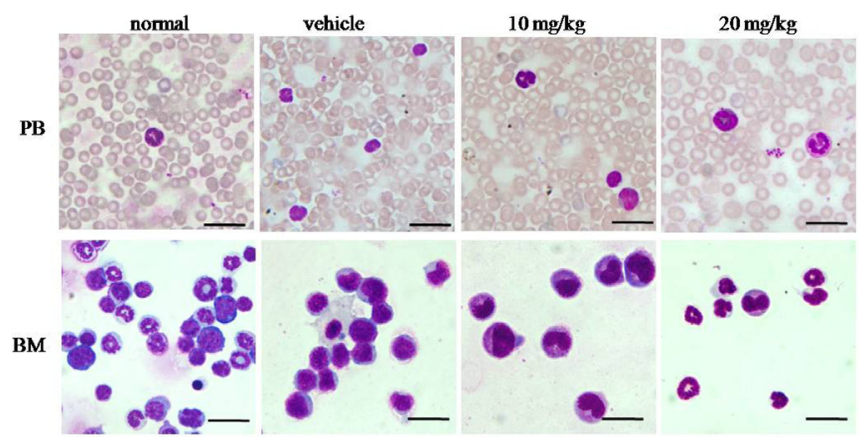

B

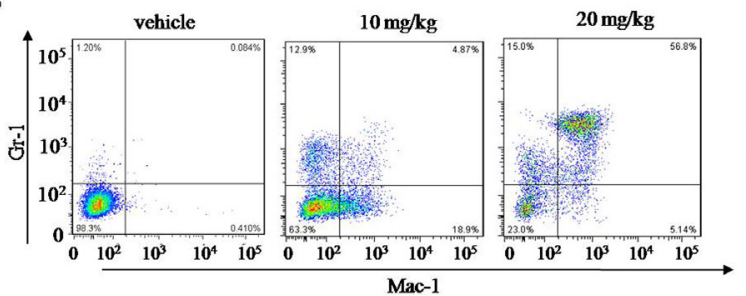

$\mathbf{C}$

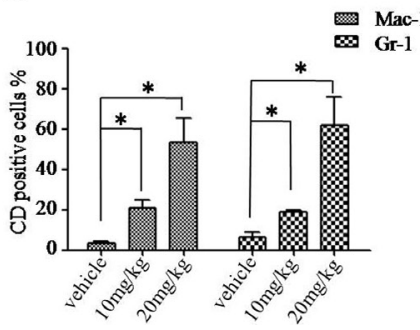

Figure 7: H7 induces leukemia-cell differentiation in vivo. APL leukemic mice were treated with vehicle and $\mathrm{H} 7$ (10 $\mathrm{mg} / \mathrm{kg}$, $20 \mathrm{mg} / \mathrm{kg}$, introperitoneal) daily for 5 consecutive days. When the first vehicle-treated leukemic mice were moribund, all mice were killed and analyzed. The cytologic analysis by Wright's staining of the peripheral blood (PB) and bone marrow (BM) are shown in (A) Scale bar, $20 \mu \mathrm{m}$. The expression levels of Gr- $1^{+}$and Mac- $1^{+}$were detected by FACS. The representative images are shown in (B) and the percentages of $\mathrm{Gr}-1^{+}$and $\mathrm{Mac}-1^{+}$are shown in $(\mathbf{C})$. All values represent the means \pm S.D. of three independent experiments. ${ }^{*} p<0.05$ compared with the vehicle-treated mice cells $(\mathrm{C})$. 
parameterized using the OPLS 2005 force field. The tautomers and ionization states expected to occur at 5.0-9.0 $\mathrm{pH}$ range were generated using the "ionize" module. During docking, the standard-precision (SP) and extra-precision (XP) dockings were adopted to generate the minimized pose, and the Glide scoring function (G-Score) was used in selecting the final pose exhibiting the lowest energy conformation for each ligand [30]. Thirty-eight compounds from the top 100 compound based on both the SP and XP scores were purchased and dissolved in DMSO for biological testing.

\section{Prdx I activity assay}

Human Prdx I was cloned into pET28a vector containing a His6 tag sequence at the N-terminal region. The proteins were expressed in the Escherichia coli strain BL21 and then purified. The Prdx I peroxidase activity was detected by a standard Trx-Trx reductase-NADPHcoupled spectrophotometric assay as described previously [20].

\section{SPR assay}

The SPR experiments were performed on Biacore T200 (GE Healthcare). Full-length human Prdx I was immobilized on a CM5 chip (GE Healthcare) aiming at 500 response units using amine coupling chemistry. Compound binding with Prdx I was analyzed in a single-cycle kinetic analysis at $0.39-25 \mathrm{mM} \mathrm{H7}$ concentration using HBS-P buffer (10 mM HEPES, pH 7.4, $150 \mathrm{mM}$ sodium chloride $(\mathrm{NaCl}), 0.05 \% \mathrm{P} 20)$ at a flow rate of $30 \mu \mathrm{l} / \mathrm{min}$ at $37^{\circ} \mathrm{C}$. Biacore T200 Evaluation Software 1.0 (GE Healthcare) was used in data analysis.

\section{CETSA}

CETSA was performed according to the method described [27]. NB4 cells were harvested and washed with PBS. The cells were diluted in PBS supplemented with complete protease inhibitor cocktail. The cell suspensions were freeze-thawed three times using liquid nitrogen, and the soluble fraction (lysate) was separated from the cell debris by centrifugation at $20,000 \mathrm{~g}$ for $20 \mathrm{~min}$ at $4^{\circ} \mathrm{C}$. The cell lysates were subsequently diluted with PBS and divided into two aliquots; one aliquot was treated with DMSO and the other with $\mathrm{H} 7$ diluent. After 10-30 min incubation at room temperature, the respective lysates were divided into smaller $(50 \mu \mathrm{L})$ aliquots and then heated individually at different temperatures for $3 \mathrm{~min}$ (Veriti Thermal Cycler, Applied Biosystems/Life Technologies) followed by cooling for $3 \mathrm{~min}$ at room temperature. The appropriate temperatures were determined in preliminary CETSA experiments (data not shown). The heated lysates were centrifuged at 20,000 g for $20 \mathrm{~min}$ at $4^{\circ} \mathrm{C}$ to separate the soluble fractions from the precipitates. The supernatants were transferred into new microtubes and then analyzed by sodium dodecyl sulfate polyacrylamide gel electrophoresis (SDS-PAGE) followed by Western blot analysis.

\section{DARTS}

DARTS is a general methodology for identifying and studying protein-ligand interactions [31] and was performed to investigate the H7-Prdx I interaction. Purified Prdx I protein were incubated with drugs or vehicle at room temperature for $50 \mathrm{~min}$, and the above mixture was digested by pronase $(10 \mathrm{mg} / \mathrm{ml}$ stock solutions in water-aliquots stored at $-20^{\circ} \mathrm{C}$ ) at appropriate ratio dissolved in TNC buffer $(50 \mathrm{mM}$ Tris- $\mathrm{HCl} \mathrm{pH}$ 8.0, $50 \mathrm{mM} \mathrm{NaCl}$, and $10 \mathrm{mM} \mathrm{CaCl}$ ) for $30 \mathrm{~min}$. The reaction was stopped by adding concentrated SDS-PAGE loading buffer to final $1 \times$, and then mixed well and boiled immediately. The samples were subjected to Western blot.

\section{Cell culture and agents}

The BM samples were collected from newly diagnosed AML patients at the Tongren Hospital and Shanghai First People's Hospital, which are affiliated to Shanghai Jiaotong University School of Medicine (SJTU-SM). Informed consent was obtained from all patients and all manipulations were approved by the Medical Science Ethics Committee of SJTU-SM. The patients were diagnosed according to the FrenchAmerican-British classification. Patient (Pt) 1 is M3 type, Pt 2-4 and 6 are M1 type, Pt 5 is M5 type. Normal cord blood $(\mathrm{CB})$ or bone marrow $(\mathrm{BM})$ samples were collected from healthy volunteers. In addition, the mononuclear cells were aspirated by Ficoll-Paque liquid. The APL cell line NB4 was obtained from Dr. Michel Lanotte (Hospital Saint Louis, Paris, France), whereas the human monocytic cell line U937 and the human myelomonocytic leukemia cell line HL60 were obtained from the American Type Culture Collection (Manassas, VA). The cells were grown in RPMI-1640 (Bio-Whittaker Europe, Verviers, Belgium) supplemented with 10\% fetal calf serum (EuroClone, Life Science Division, Milan, Italy) at $37^{\circ} \mathrm{C}$ in a humidified atmosphere of $5 \% \mathrm{CO}_{2}$.

\section{Cell differentiation assay}

The morphological features of the cells were examined by microscopy after Wright's staining (BASO Diagnostic Inc., Guangdong, China) and the cell surface differentiation antigens CD11b and CD14 were measured using fluorescein isothiocyanate- or phycoerythrin-labeled antibodies with isotype controls (Beckman-Coulter, Miami, FL) by flow cytometry. 


\section{Detection of intracellular ROS}

The oxidation-sensitive fluorescent probe dye 2', 7'-dichlorodihydrofluorescein diacetate (DCF-DA, Invitrogen Molecular Probes, Eugene, OR) was used in measuring the intracellular ROS. DCF-DA is deacetylated intracellularly by nonspecific esterases and is further oxidized by cellular peroxides to the fluorescent compound 2', 7'-dichlorofluorescein. Briefly, the cells treated with or without $\mathrm{H} 7$ were washed with phosphate buffered saline (PBS) and then incubated with $20 \mu \mathrm{M}$ DCF-DA at $37^{\circ} \mathrm{C}$ for $30 \mathrm{~min}$ according to the manufacturer's instructions. The fluorescence signals were detected by a FACStar Flow Cytometer (Beckman Coulter). For each sample, 5,000 or 10,000 events were collected. In addition, the $\mathrm{H}_{2} \mathrm{O}_{2}$ levels were expressed in terms of mean fluorescence intensity.

\section{RNA interference and transfection}

Pairs of complementary oligonucleotides against AKT (5'-GTGGTCATGTACGAGATGA-3'), C/EBP $\beta$ [20], and non-target control shRNA were synthesized by Sangon Biotech (Shanghai), annealed, and ligated into the PSIREN-RetroQ Vector (Clontech Laboratories). These shRNA-carrying retroviruses, which were produced in 293T cells, were used to infect the NB4 cells.

\section{Western blot}

Equal volumes of cell lysates were loaded onto $10 \%$ SDS-polyacrylamide gel, electrophoresed, and then transferred into ECL-nitrocellulose membranes (Amersham, Buckinghamshire, UK). The membranes were stained with $0.2 \%$ Ponceau $\mathrm{S}$ red to ensure equal protein loading. After blocking with 5\% nonfat milk in Tris-buffered saline, the membranes were incubated with the polyclonal antibodies against Prdxs I-V, C/EBPa, and C/EBP $\beta$ (Santa Cruz Biotechnology, Santa Cruz, CA) overnight at $4^{\circ} \mathrm{C}$ followed by incubation with horseradish peroxidase-linked secondary antibody (cell signaling) for $1 \mathrm{~h}$ at room temperature. Detection was performed by SuperSignal West Pico Chemiluminescent Substrate kit (Pierce, Rockford, IL) according to manufacturer's instruction. $\beta$-actin (Merck, Darmstadt, Germany) was used as an internal control. Signal intensity of proteins was normalized against $\beta$-actin using Quantity One (Bio-Rad).

\section{Mouse APL model}

The leukemia-cells isolated from the spleens of leukemic hMRP8-PML-RARa transgenic mice were intravenously injected into the 6- to 8-week-old female $\mathrm{FVB} / \mathrm{N}$ mice following sublethal irradiation. Three days after transplantation, the mice were intraperitoneally treated with vehicle (15\% Cremophor EL, 15\% Propanediol, and $60 \%$ PBS) or H7. Cytological and histological analyses were also performed. The $\mathrm{Gr}^{-1}{ }^{+} /$ Mac- $1^{+}$cells in BM were determined by FACS. Animal handling was approved by the Committee for Humane Treatment of Animals of SJTU-SM.

\section{Statistical analysis}

Student's $t$-test was used in evaluating the difference between two treatments. A $p$ value of less than 0.05 was considered statistically significant.

\section{ACKNOWLEDGMENTS AND FUNDING}

This work was supported in part by the grants from the National Basic Research Program of China (973 Program) (No. 2015CB910403 and 2013CB910903), National Natural Science Foundation of China (91313303, 81272886, 81300404, 81570118, 31100980, and 31300679), Science and Technology Committee of Shanghai (11JC1406500, 15401901800, 13431900501, and 13ZR1456900), and Shanghai Talent Development Project of Shanghai Human Resource and Social Security Bureau.

\section{Abbreviations}

Prdx I, peroxiredoxin I; ATRA, all trans-retinoic acid; ROS, reactive oxygen species; NAC, N-acetyl cysteine; APL, acute promyelocytic leukemia; PBS, phosphate buffered saline; PDB, Protein Data Bank; RMSD, rootmean-square deviation; SP, standard-precision; XP, extraprecision; G-Score, Glide scoring function; CETSA, Cellular Thermal Shift Assay; GPx, glutathione peroxidase 1.

\section{CONFLICTS OF INTEREST}

The authors declare no conflicts of interest.

\section{REFERENCES}

1. Gocek E, Marcinkowska E. Differentiation therapy of acute myeloid leukemia. Cancers (Basel). 2011; 3:2402-2420.

2. Hughes PJ, Marcinkowska E, Gocek E, Studzinski GP, Brown G. Vitamin D3-driven signals for myeloid cell differentiation-implications for differentiation therapy. Leuk Res. 2010; 34:553-565.

3. Marchwicka A, Cebrat M, Sampath P, Sniezewski L, Marcinkowska E. Perspectives of differentiation therapies of acute myeloid leukemia: the search for the molecular basis of patients' variable responses to 1,25-dihydroxyvitamin $d$ and vitamin d analogs. Front Oncol. 2014; 4:125.

4. Trump DL, Deeb KK, Johnson CS. Vitamin D: considerations in the continued development as an agent for cancer prevention and therapy. Cancer J. 2010; 16:1-9. 
5. Hole PS, Darley RL, Tonks A. Do reactive oxygen species play a role in myeloid leukemias? Blood. 2011; 117:5816-5826.

6. Yamamoto T, Sakaguchi N, Hachiya M, Nakayama F, Yamakawa M, Akashi M. Role of catalase in monocytic differentiation of U937 cells by TPA: hydrogen peroxide as a second messenger. Leukemia. 2009; 23:761-769.

7. Irwin ME, Rivera-Del Valle N, Chandra J. Redox control of leukemia: from molecular mechanisms to therapeutic opportunities. Antioxid Redox Signal. 2013; 18:1349-1383.

8. Owusu-Ansah E, Banerjee U. Reactive oxygen species prime Drosophila haematopoietic progenitors for differentiation. Nature. 2009; 461:537-541.

9. Noh EK, Kim H, Park MJ, Baek JH, Park JH, Cha SJ, Won JH, Min YJ. Gefitinib enhances arsenic trioxide (AS2O3)-induced differentiation of acute promyelocytic leukemia cell line. Leuk Res. 2010; 34:1501-1505.

10. Liu T, Men Q, Wu G, Yu C, Huang Z, Liu X, Li W. Tetrandrine induces autophagy and differentiation by activating ROS and Notch1 signaling in leukemia cells. Oncotarget. 2015. doi: 10.18632/oncotarget.3505.

11. Rhee SG, Woo HA. Multiple functions of peroxiredoxins: peroxidases, sensors and regulators of the intracellular messenger $\mathrm{H}(2) \mathrm{O}(2)$, and protein chaperones. Antioxid Redox Signal. 2011; 15:781-794.

12. Ray PD, Huang BW, Tsuji Y. Reactive oxygen species (ROS) homeostasis and redox regulation in cellular signaling. Cell Signal. 2012; 24:981-990.

13. Park J, Lee S, Kang SW. 2-cys peroxiredoxins: emerging hubs determining redox dependency of Mammalian signaling networks. Int J Cell Biol. 2014; 2014:715867.

14. Woo HA, Yim SH, Shin DH, Kang D, Yu DY, Rhee SG. Inactivation of peroxiredoxin I by phosphorylation allows localized $\mathrm{H}(2) \mathrm{O}(2)$ accumulation for cell signaling. Cell. 2010; 140:517-528.

15. Demasi AP, Ceratti D, Furuse C, Cury P, Junqueira JL, Araujo VC. Expression of peroxiredoxin I in plasma cells of oral inflammatory diseases. Eur J Oral Sci. 2007; 115:334-337.

16. Demasi AP, Magalhaes MH, Furuse C, Araujo NS, Junqueira JL, Araujo VC. Peroxiredoxin I is differentially expressed in multiple myelomas and in plasmablastic lymphomas. Oral Dis. 2008; 14:741-746.

17. Chhipa RR, Lee KS, Onate S, Wu Y, Ip C. Prx1 enhances androgen receptor function in prostate cancer cells by increasing receptor affinity to dihydrotestosterone. Mol Cancer Res. 2009; 7:1543-1552.

18. Zhang B, Wang Y, Su Y. Peroxiredoxins, a novel target in cancer radiotherapy. Cancer Lett. 2009; 286:154-160.

19. Riddell JR, Bshara W, Moser MT, Spernyak JA, Foster BA, Gollnick SO. Peroxiredoxin 1 controls prostate cancer growth through Toll-like receptor 4-dependent regulation of tumor vasculature. Cancer Res. 2011; 71:1637-1646.
20. Liu CX, Yin QQ, Zhou HC, Wu YL, Pu JX, Xia L, Liu W, Huang X, Jiang T, Wu MX, He LC, Zhao YX, Wang XL, et al. Adenanthin targets peroxiredoxin I and II to induce differentiation of leukemic cells. Nat Chem Biol. 2012; 8:486-493.

21. Liu CX, Zhou HC, Yin QQ, Wu YL, Chen GQ. Targeting peroxiredoxins against leukemia. Exp Cell Res. 2013; 319:170-176.

22. Martinez Molina D, Jafari R, Ignatushchenko M, Seki T, Larsson EA, Dan C, Sreekumar L, Cao Y, Nordlund P. Monitoring drug target engagement in cells and tissues using the cellular thermal shift assay. Science. 2013; 341:84-87.

23. Wang R, Xia L, Gabrilove JL, Waxman S, Jing Y. Sorafenib inhibition of Mcl-1 accelerates ATRA induced apoptosis in differentiation responsive AML cells. Clin Cancer Res. 2015 Oct 12; PMID: 26459180.

24. Imran M, Park TJ, Lim IK. TIS21/BTG2/PC3 enhances downregulation of c-Myc during differentiation of HL-60 cells by activating Erk1/2 and inhibiting Akt in response to all-trans-retinoic acid. Eur J Cancer. 2012; 48:2474-2485.

25. Huber R, Pietsch D, Panterodt T, Brand K. Regulation of C/ EBPbeta and resulting functions in cells of the monocytic lineage. Cell Signal. 2012; 24:1287-1296.

26. Ding Q, Jin T, Wang Z, Chen Y. Catalase potentiates retinoic acid-induced THP-1 monocyte differentiation into macrophage through inhibition of peroxisome proliferator-activated receptor gamma. J Leukoc Biol. 2007; 81:1568-1576.

27. Jafari R, Almqvist $H$, Axelsson $H$, Ignatushchenko $M$, Lundback T, Nordlund P, Martinez Molina D. The cellular thermal shift assay for evaluating drug target interactions in cells. Nat Protoc. 2014; 9:2100-2122.

28. Wen CL, Teng CL, Chiang CH, Chang CC, Hwang WL, Kuo CL, Hsu SL. Methanol extract of Antrodia cinnamomea mycelia induces phenotypic and functional differentiation of HL60 into monocyte-like cells via an ERK/CEBP-beta signaling pathway. Phytomedicine. 2012; 19:424-435.

29. Ji Y, Lee HJ, Goodman C, Uskokovic M, Liby K, Sporn M, Suh N. The synthetic triterpenoid CDDO-imidazolide induces monocytic differentiation by activating the Smad and ERK signaling pathways in HL60 leukemia cells. Mol Cancer Ther. 2006; 5:1452-1458.

30. Huang Z, Mou L, Shen Q, Lu S, Li C, Liu X, Wang G, Li S, Geng L, Liu Y, Wu J, Chen G, Zhang J. ASD v2.0: updated content and novel features focusing on allosteric regulation. Nucleic Acids Res. 2014; 42:D510-516.

31. Lomenick B, Olsen RW, Huang J. Identification of direct protein targets of small molecules. ACS Chem Biol. 2011; 6:34-46. 